\title{
Cardiopulmonary Arrest Caused by Acute Myocardial Infarction with Multivessel Coronary Disease in a Young Adult with Von Recklinghausen's Disease
}

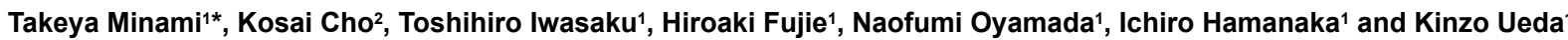

${ }^{1}$ Rakuwakai Kyoto Cardiovascular Intervention Center, Rakuwakai Marutamachi Hospital, Kyoto, Japan

${ }^{2}$ Department of Primary Care and Emergency Medicine, Kyoto University Graduate School of Medicine, Kyoto, Japan

\begin{abstract}
The case involved a 38-year-old man. By 30-year-old, he had undergone surgical resection of schwannoma four times and had been diagnosed with von Recklinghausen's disease (neurofibromatosis type I; NF1). At 35 years old, invasion of the thoracic vertebral bodies due to NF1 was recognized along with exertional chest and back pain. In November 2014, he collapsed suddenly while walking and was urgently transported to our emergency room after the return of spontaneous circulation. Forrester subset IV heart failure was recognized, and chronic total occlusion was observed in both the right coronary and left circumflex arteries, while the culprit lesion for acute coronary syndrome was in the proximal left anterior descending artery (LAD). During emergent percutaneous coronary intervention $(\mathrm{PCl})$ to the $L A D$, revascularization was difficult due to slow flow caused by unexpected multiple thrombi that disappeared immediately after argatroban injection. Although we finished $\mathrm{PCl}$ with thrombolysis in myocardial infarction grade 3 flow in the LAD, we could not save the patient because of worsening traumatic brain hemorrhage and decreased left ventricular function. Autopsy showed no subacute thrombosis of the drug-eluting stent deployed in the LAD. Autopsy also revealed severe atherosclerosis of multiple vessels, including the coronary vessels, representing a rare finding in typical NF1 patients.
\end{abstract}

Keywords: Von Recklinghausen's disease Neurofibromatosis 1(neurofibromatosis type I; NF1); Percutaneous coronary intervention (PCI); Cardiopulmonary arrest; Acute Coronary Syndrome(ACS); Argatroban

\section{Introduction}

Neurofibromatosis represents a group of autosomal-dominant disorders with two distinct forms: neurofibromatosis type 1 (NF1; von Recklinghausen's disease); and neurofibromatosis type $2[1,2]$. Diagnosis of NF1 is based on clinical features such as café-au-lait spots or neurofibroma [3]. The NF1 gene encodes the neurofibromin protein, which plays a role in tumor suppression by inactivation of Ras-mitogen-activated protein kinase (MAPK) pathway. Inactivation of the NF1 gene by mutation or allelic loss leads to tumor-like cell proliferation of several tissues in the disease with aging [4]. Coronary artery disease in NF1 patients is quite rare, although vascular disease such as renal hypertension, abdominal aortic coarctation, or aneurysms are relatively well-recognized [5]. We report a case of NF1 complicated by severe coronary artery disease, and describe the results of autopsy with reference to the literature.

\section{Case Report}

The patient was a 38-year-old Japanese man. By 30 years old, he had undergone surgical resection of schwannoma four times and was diagnosed with NF1. At 35 years old, invasion of the thoracic vertebral bodies due to NF1 was recognized along with exertional chest and back pain. In November 2014, he collapsed suddenly while walking. A passerby called emergency medical services without providing bystander cardiopulmonary resuscitation. The patient was transported to our emergency room with the return of spontaneous circulation after a total of $21 \mathrm{~min}$ in cardiac arrest. Ventricular fibrillation (VF) had been treated using direct current shocks twice. In the emergency room, Glasgow Coma Scale score was E1VTM1. Blood pressure was 149/99 $\mathrm{mmHg}$ and heart rate was 100 beats/min under a norepinephrine dose of $0.2 \mu \mathrm{g} / \mathrm{kg}$ body weight $/ \mathrm{min}$. Computed tomography (CT) of the head showed traumatic contusion in the right intracranial temporal region with a small volume of hemorrhage. Multiple café-au-lait spots were evident on the skin. Blood test showed untreated dyslipidemia and elevated concentrations of cardiac enzymes. Electrocardiography (ECG) showed ST elevation in I, II, III, aVF and $\mathrm{V}_{2-5}$, abnormal Q waves in II, III and aVF and ST depression in aVR (Figures 1a-1c). Echocardiography revealed moderate mitral regurgitation (MR) and decreased motion of the left ventricular wall (ejection fraction (EF), 40\%) with akinesis from the base to the apical portion of the anterior wall and akinesis of the middle to apical portion of the lateral and inferior wall, with these areas appearing hyperechoic. After diagnosis of ST-segment elevation myocardial infarction (STEMI) with comatose post-cardiac arrest syndrome, we performed emergent cardiac catheterization with target temperature management using an external-cooling device (Arctic Sun Temperature Management System; Medivance, Louisville, CO). The Swan-Ganz catheter showed acute heart failure (Forrester subset 4: cardiac index, $2.13 \mathrm{~L} / \mathrm{min} / \mathrm{m}^{2}$; pulmonary capillary wedge pressure, $35 \mathrm{mmHg}$ ). Left ventriculography showed decreased EF with focal asynergy and Grade $2 \mathrm{MR}$, supporting the findings from echocardiography.

After administration of heparin, control coronary angiography revealed culprit lesions of acute coronary syndrome (ACS) in the proximal part of the left anterior descending artery (LAD) with thrombolysis in myocardial infarction (TIMI) grade 2 flow and chronic

*Corresponding author: Takeya Minami, Rakuwakai Kyoto Cardiovascular Intervention Center, Rakuwakai Marutamachi Hospital, Shitihonmatsu Marutamachi Agaru, Nakagyo-ku, Kyoto 604-8405, Kyoto, Japan, Tel: 81758010351; Fax: 81758015571; E-mail: tminami@kuhp.kyoto-u.ac.jp

Received December 04, 2017; Accepted December 11, 2017; Published December 14, 2017

Citation: Minami T, Cho K, Iwasaku T, Fujie H, Oyamada N, et al. (2017) Cardiopulmonary Arrest Caused by Acute Myocardial Infarction with Multivesse Coronary Disease in a Young Adult with Von Recklinghausen's Disease. J Cardiovasc Dis Diagn 6: 303. doi: 10.4172/2329-9517.1000303

Copyright: ( 2017 Minami T, et al.. This is an open-access article distributed under the terms of the Creative Commons Attribution License, which permits unrestricted use, distribution, and reproduction in any medium, provided the original author and source are credited. 

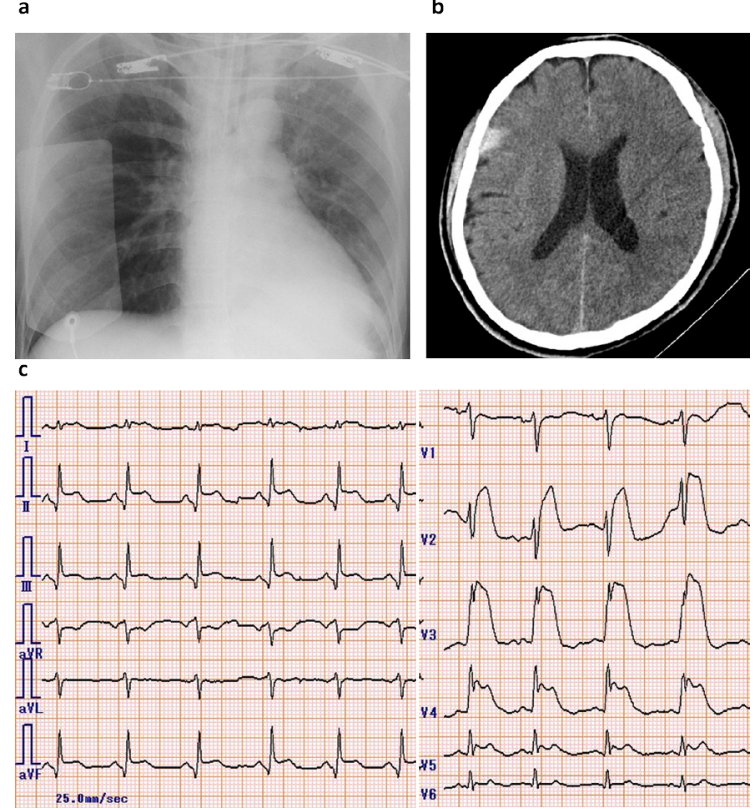

Figure 1: Findings on admission. a) Chest $X$-ray shows butterfly shadow and cardiomegaly. b) Computed tomography of the head reveals traumatic cerebral contusion. c) Electrocardiography shows a heart rate of 90 beats/min, QTc of $0.45 \mathrm{~s}$, ST elevation in I, II, III, aVF and $\mathrm{V}_{2-5}$, abnormal Q waves in II, III and aVF and ST depression in aVR.
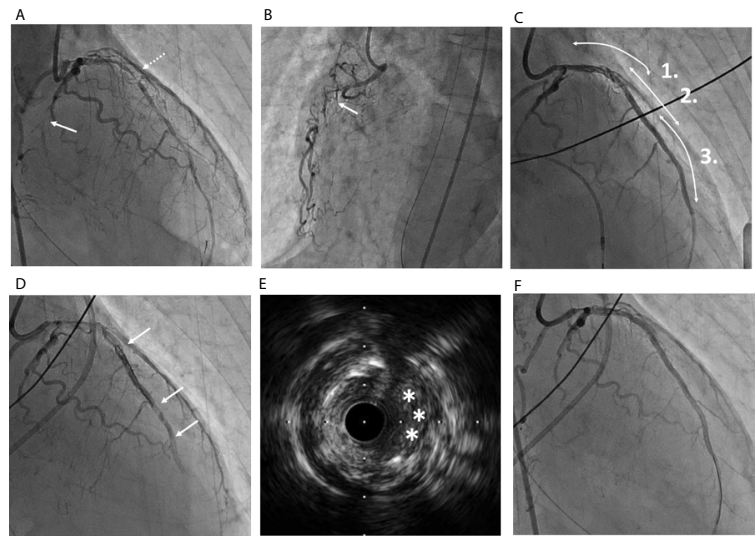

Figure 2: Results of angiography and Intravascular ultrasonography (IVUS) a) Left coronary artery (LCA) control angiogram (right anterior oblique craniocaudal view). White arrow shows chronic total occlusion (CTO) of the left circumflex artery (LCX); white dotted arrow shows the culprit lesion causing ACS in the LAD. b) Right coronary artery (RCA) control angiogram (left anterior oblique). White arrow shows CTO of the RCA. The LCA is the dominant coronary artery. The RCA shows a high anterior origin. c) Three drug-eluting stents were deployed in the LAD. 1) Resolute integrity, 3.0-30 mm; 2) Promus premier 2.5-38 mm; 3) Promus premier 2.25-32 mm. d) White arrows show multiple thrombi. e) IVUS shows multiple thrombi (asterisk). f) After argatroban injection.

total occlusion (CTO) in both the middle part of the right coronary artery (RCA) and the distal part of the left circumflex artery (LCX) in the left dominant coronary arteries with an anomalous high-anterior origin of the RCA. The entirety of the left ventricular myocardium was jeopardized, while collateral feeding was seen from the LAD to both the RCA and LCX (Figures 2a-2f). Because VF/pulseless electrical activity relapsed sporadically, we decided to perform emergent PCI to the LAD to improve hemodynamics using femoral approach. A 7-Fr JL4.0SH guiding catheter was introduced. After crossing the culprit lesion with a 0.014 " Sion blue guidewire (Asahi Intec, Nagoya, Japan), red thrombus was aspirated through the aspiration catheter (Thrombuster III GR; Kaneka, Osaka, Japan). Intravascular ultrasonography (IVUS) (Terumo, Tokyo, Japan) showed severe stenosis with lipid-rich plaque in the proximal LAD. We placed the stent (Resolute Integrity $3.0-30 \mathrm{~mm}$ R-ZES; Medtronic, Santa Rosa, CA) in the proximal part of the LAD with a distal protection device (Filtrap; Nipro, Tokyo, Japan). Slow-flow phenomenon and VF relapsed even after retrieving the distal protection device and aspiration using Thrombuster III GR with nicorandil and heparin under conditions of prolonged activated clotting time (278 s). Although we added two more stents (Promus Premier 2.5-38 mm + 2.25-32 mm; Boston Scientific, Natick, MA) in the middle to distal part of the LAD with intra-aortic balloon pumping (IABP) (to minimize the size of the myocardial infarct and prevent subacute stent thrombosis (SAT) ) and provided temporary pacing support, maintaining LAD flow was difficult due to multiple unexpected recurrent thrombi identified on IVUS (Figure 2). After administration of argatroban ( $3 \mathrm{mg}$ bolus into the LAD), the unexpected thrombi rapidly disappeared (Figure 2). We successfully completed PCI with TIMI grade 3 flow in the LAD. Despite intensive care with IABP and a full dose of vasopressor, maintaining blood pressure proved difficult. CT of the head revealed worsening of the brain contusion and hemorrhage. The patient was declared dead $9 \mathrm{~h}$ after admission. No sign of SAT or cardiac rupture was evident from ECG or echocardiography at follow-up. Heparininduced thrombocytopenia (HIT) antibody was negative and no abnormal findings were identified for lupus anticoagulant protein $\mathrm{C}$ (antigen and activity), protein S (total and free), anticardiolipin or antiphosphatidylserine antibodies. Autopsy revealed that none of the stents in the LAD were occluded and the LAD remained patent (Figures 3a-3f). Proliferation of spindle-shaped smooth muscle cells and detachment of the media were seen in all coronary arteries that had shown chronic total occlusion. These lesions were not seen in large vessels like the aorta, but were characteristically found in small to medium-sized vessels such as the coronary arteries and splenic artery (Figure 3).

\section{Discussion}

We experienced a case involving a young adult with NF1 who developed ACS in the form of STEMI. We were unable to rescue this young man. This was because severe heart failure is hard to treat when caused by ACS involving the LAD as the only remaining circulation due to severe multivessel coronary disease (MVD) including CTO of the RCA and LCX, and because coronary arteries with unexpected multiple thrombi are difficult to revascularize. Chronic and severe MVD were seen despite the young age of the patient. Our first question was thus whether any cases of NF1 exhibiting complications of early coronary artery disease had been described. Although cardiovascular complications in medium-sized to large vessels such as aortic coarctation, aortic aneurysm and renal artery stenosis are well-known complications in NF1 patients, coronary artery disease is a rare but definite possibility in NF1 patients [6]. A small number of reports have described young adults with NF1 who died due to acute coronary syndrome (ACS) and early MVD, showing similarity to our case in terms of both age and severity [7]. Patients with NF1 develop neoplastic lesions in various organs that progress with age due to abnormal cell proliferation caused by a mutation in NF1. These lesions can therefore develop in small to medium-sized vessels such as the coronary arteries from a young age without the more generalized risk factors of atherosclerosis such as dyslipidemia, hypertension, smoking, and aging. When a patient with NF1 experiences chest pain, 

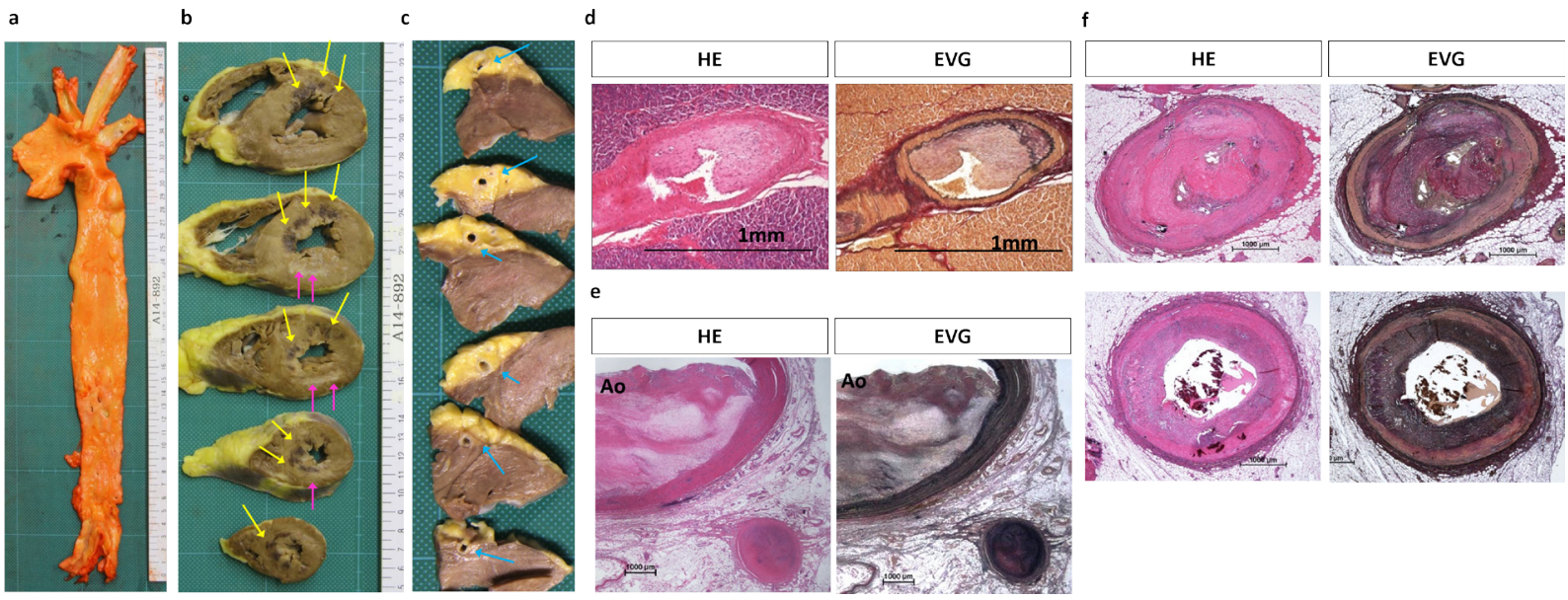

Figure 3: Macro- and microscopic images from autopsy. a) Aorta (longitudinal) shows mild atherosclerosis, with total occlusion of small to medium-sized vessels. b) LV serial section (short axis): sub-endocardial infarction is revealed in the antero-septal wall, with old infarction seen in the inferior wall. c) Serial LAD sections (short axis) show no sites of occlusion in the LAD stent. d) Serial microscopic images of the pancreatic artery. e) Serial microscopic images of the aorta. No significant atherosclerosis of large vessels is apparent. Small vessels appear obstructed by proliferated neointima. f) Autopsy micro image of coronary arteries. Atherosclerotic lesions are severe in the coronary arteries, including occlusion. HE, hematoxylin and eosin; EVG, Elastica van Gieson. Proliferation of spindle-shaped or angioma-like intima and detachment of media are apparent.

potential causes should be assessed not only by considering common complications such as spinal deformities but also by evaluating the coronary arteries, even if the patient is young or attends clinical departments other than cardiology, such as dermatology. In the present case, we encountered multiple thrombi during intervention. This led to a second question: is there any interaction between NF1 and HIT? The NF1 gene is also expressed in platelets. One report concerning NF1 showed that an attenuated platelet response to von Willebrand factor and tissue factors results in a tendency to bleed and that irregular antibodies to the coagulation system are observed at the same time [8]. We postulated that thrombosis based on an immune mechanism similar to the interactions between NF1 and HIT may have been involved. Our strategy has been to stop using heparin and immediately administer argatroban when unexpected thrombus is found during intervention. We implemented this strategy because thrombi that appear during interventions are serious and because we have frequently experienced cases in which we were able to save the patient through the use of argatroban based on prioritizing treatment of the patient over definitive diagnosis of HIT based on test results. In the present case, use of argatroban proved useful in addressing the unexpected thrombi during intervention, and autopsy imaging revealed the absence of occlusion in stents placed at the LAD.

\section{Conclusion}

We have reported the case of a young patient with NF1 and MVD. If angina is encountered in a von Recklinghausen's patient (even if young), aggressive evaluation of the coronary arteries and early intervention should be considered. Argatroban effectively resolved unexpected thrombus in coronary arteries during intervention in this case.

\section{Acknowledgements}

We wish to thank Dr. T. Sugimoto from the Cardiovascular Intervention Center at Marutamachi Hospital, and Dr. H. Yasui from the Department of Pathology at Otowa Hospital for their clinical support.

\section{Disclosure}

The authors have no conflicts of interest to declare. Informed consent for publication of this case was obtained from the family of the patient.

\section{References}

1. Gutmann DH, Aylsworth A, Carey JC, Korf B, Marks J, et al. (1997) The diagnostic evaluation and multidisciplinary management of neurofibromatosis 1 and neurofibromatosis 2. JAMA 278: 51-57.

2. Boyd KP, Korf BR, Theos A (2009) Neurofibromatosis type 1. J Am Acad Dermatol 61: 1-14.

3. Reynolds RM, Browning GG, Nawroz I, Campbell IW (2003) Von Recklinghausen's neurofibromatosis: Neurofibromatosis type 1. Lancet 361 1552-1554.

4. Li Y, O'Connell P, Breidenbach HH, Cawthon R, Stevens J, et al. (1995) Genomic organization of the neurofibromatosis 1 gene (NF1). Genomics 25: 9-18.

5. Oderich GS, Sullivan TM, Bower TC, Gloviczki P, Miller DV, et al. (2007) Vascular abnormalities in patients with neurofibromatosis syndrome type I: clinical spectrum, management, and results. J Vasc Surg 46: 475-484

6. Friedman JM, Arbiser J, Epstein JA, Gutmann DH, Huot SJ, et al. (2002) Cardiovascular disease in neurofibromatosis 1: Report of the NF1 cardiovascular task force. Genet Med 4: 105-111.

7. Lie JT (1998) Vasculopathies of neurofibromatosis type 1 (von Recklinghausen disease). Cardiovasc Pathol 7: 97-108.

8. Rasko JE, North KN, Favaloro EJ, Grispo L, Berndt MC (1995) Attenuated platelet sensitivity to collagen in patients with neurofibromatosis type $1 . \mathrm{Br} J$ Haematol 89: 582-588. 